\title{
FLOWS THROUGH NONHOMOGENEOUS POROUS MEDIA IN AN ISOLATED ENVIRONMENT
}

\author{
BY \\ JINGXUE YIN AND WENJIE GAO \\ Department of Mathematics, Jilin University, P. R. China
}

\begin{abstract}
The nonhomogeneous gas or fluid flowing through a nonhomogeneous porous medium is studied. An interesting phenomenon is discussed which shows that the state of the flow is not affected by the surrounding environment if some hypotheses are made on the porosity of the gas, the viscosity, and the permeability of the medium. Such a state is called an isolated environment. The conditions under which the state of the gas depends on the surrounding environment are also discussed.
\end{abstract}

1. Introduction. Consider a nonhomogeneous gas (or fluid) flowing through a nonhomogeneous porous medium. Let $u=u(x, t)$ represent the density of the gas at any point $(x, t)$ where $x$ is the position variable, $t$ the time variable, and $p$ the pressure. If the flow is isothermic then

$$
u=\gamma_{0} p
$$

for some constant $\gamma_{0}$. Dynamically, the motion is characterized by conservation of mass,

$$
\operatorname{div}(u v)=-f \frac{\partial u}{\partial t}
$$

where $f$ is the porosity of the medium and $v$ the velocity vector. By Darcy's law,

$$
v=-\frac{\kappa}{\mu} \nabla p
$$

where $\mu$ is the viscosity of the gas and $\kappa$ the permeability of the medium.

Since the gas is nonhomogeneous and the medium is also nonhomogeneous, we know that $\kappa$ and $\mu$ may depend on the density of the gas, the position, the pressure, and even the rate of change of the density and the pressure. Furthermore, $f$ may be a function of the position.

Combining the above equations, we obtain

$$
f \frac{\partial u}{\partial t}=\operatorname{div}\left(\frac{\kappa \gamma_{0} u}{\mu} \nabla u\right)=\operatorname{div}(\phi(x, t, u, \nabla u) \nabla u) .
$$

Received September 6, 1994.

1991 Mathematics Subject Classification. Primary 35K55, 35K65, 35Q30. 
Many results have been obtained in recent years involving such equations. There are numerous papers studying the nonstationary filtration in partially saturated porous media, see $[1-5,7-12]$ and the references therein. The important examples are the equations of Newtonian polytropic filtration $[1-4,7-8]$ and the non-Newtonian elastic filtration $[5,9-11]$.

The nonstationary flow in a porous medium of fluids with a power dependence of the tangential stress on the velocity of the displacement under elastic conditions is described by the equation of non-Newtonian elastic filtration

$$
\frac{\partial u}{\partial t}=\operatorname{div}\left(|\nabla u|^{p-1} \nabla u\right) \quad(p=\text { constant }>0) .
$$

In the case of a more complicated dependence between the stresses and the velocities of the displacement, one must consider equations with non-power nonlinearities.

In this paper, we restrict ourselves to the discussion of the case when $\phi$ is independent of $u$ and $t$. Furthermore, we may write $\phi(x, \nabla u)=\phi(x) \psi(\nabla u)$ if we enforce the reasonable assumption that the viscosity of the gas is proportional to the density at any point and the permeability is a function of the rate of change of the pressure.

We will take the equation of non-Newtonian elastic filtration as an important example in illustrating our results. It is not hard to see that our results can also be applied to the case when we replace $u$ in the equation by a function of $u, A(u)$, for $A(s)$ satisfying some smoothness and monotonicity conditions. We will not present the details in this paper.

We consider the model in the unit ball $B$ of $\mathbf{R}^{n}$ for $n \geq 1$ and assume that the functions $\varphi, \psi$, and $f$ are all radially symmetric with respect to their variables. Mathematically, the model is in the following form:

$$
f(|x|) \frac{\partial u}{\partial t}=\operatorname{div}(\varphi(1-|x|) \psi(|\nabla u|) \nabla u), \quad \text { for } x \in B
$$

with $f>0$ in $B$ and $\psi(s)>0$ for $s>0$. Another peculiarity of the model is that it may degenerate when $\varphi(0)=0, f(1)=0$, or $\psi(0)=0$.

Denote

$$
\Psi(s)=\psi(|s|) s
$$

Obviously, $\Psi$ is an odd function.

To simplify the problem further, we introduce the polar coordinate system. Then the original equation becomes

$$
\begin{aligned}
f(r) \frac{\partial u}{\partial t} & =\sum_{i=1}^{n} \frac{\partial}{\partial x_{i}}\left(\varphi(1-r) \psi\left(\left|\frac{\partial u}{\partial r}\right|\right) \frac{\partial u}{\partial r} \cdot \frac{x_{i}}{r}\right) \\
& =\frac{\partial}{\partial r}\left(\varphi(1-r) \psi\left(\left|\frac{\partial u}{\partial r}\right|\right) \frac{\partial u}{\partial r}\right)+\frac{n-1}{r} \varphi(1-r) \psi\left(\left|\frac{\partial u}{\partial r}\right|\right) \frac{\partial u}{\partial r}
\end{aligned}
$$

Multiplying the equation by $r^{n-1}$, we have

$$
f(r) \frac{\partial\left(r^{n-1} u\right)}{\partial t}=\frac{\partial}{\partial r}\left(r^{n-1} \varphi(1-r) \psi\left(\left|\frac{\partial u}{\partial r}\right|\right) \frac{\partial u}{\partial r}\right) .
$$


We may always assume the initial state

$$
\left.u\right|_{t=0}=u_{0}(r)
$$

in studying the behavior of the gas or the fluid.

Since we are treating radial solutions in the ball $B$ for Eq. (1.1), the natural boundary value condition at $r=0$ should be

$$
\left.\frac{\partial u}{\partial r}\right|_{r=0}=0
$$

which implies the zero flux condition

$$
\left.\left.J\right|_{r=0} \equiv r^{n-1} \varphi(1-r) \psi\left(\left|\frac{\partial u}{\partial r}\right|\right) \frac{\partial u}{\partial r}\right|_{r=0}=0 .
$$

Therefore, Eq. (1.1) is now reduced to Eq. (1.2) and the suitable initial and boundary conditions are (1.3) and (1.4). To determine the state of the flow, we sometimes need to know the behavior of the flow on the set $r=1$. Our results show that whether the flow depends on the boundary behavior is determined by the viscosity of the gas and the permeability of the medium.

In this paper, we are interested in the case when the permeability of the medium is allowed to approach 0 at the boundary of the region and we can even consider the case when the porosity of the medium approaches zero at the boundary of the region. We are mainly concerned with two interesting phenomena. The first is if there are some constraints to the permeability and/or to the porosity of the medium so that the flow is uniquely determined by the initial state without interference from the boundary states. This is an interesting phenomenon that we call an isolated environment since the state is not affected by the outside environment. The second is whether there are some conditions under which there exists a uniquely determined state $u$ for any given boundary status at $r=1$ and initial state. This implies that the surrounding environment determines the state of the gas or the fluid.

The above two states exist under some conditions on the permeability of the medium and the viscosity of the gas. In fact, the integral

$$
I(\lambda)=\int_{0}^{\frac{1}{2}} \Psi^{-1}\left(\frac{\lambda}{\varphi(r)}\right) d r, \quad \lambda>0,
$$

plays an important role in determining the states, where $\Psi^{-1}$ is the inverse function of $\Psi$ whose existence follows from the monotonicity.

It is worth pointing out that if we consider the equation of non-Newtonian elastic filtration $(\mathrm{N}), I(\lambda)<\infty$ for any $\lambda>0$. The result in Sec. 2 implies that there is no isolated environment for such an equation.

We shall present the conditions and conclusions in the isolated environment in the next section and give some answers to the question of existence and uniqueness of the states depending on the boundary behavior of the flow in the last section. 
2. An isolated environment. In this section, we show an interesting phenomenon of the flow. That is, if the integral $I(\lambda)=\infty$ for any positive $\lambda$, then the flow will be determined uniquely by the initial state and the surrounding environment will have no effect on the state.

Throughout the paper, we shall use $C_{*}^{1,1 / 2}\left(Q_{T}\right)$ to denote the set of functions that are locally Lipschitz continuous with respect to $r$ in $(0,1)$ and Hölder continuous in $t$ with exponent $\frac{1}{2}$ in $[0, T]$. We will always adopt the following hypotheses:

1. $\Psi \in C^{1}, \Psi(s)$ is monotonically increasing in its variable $s$ and $\Psi(s) \rightarrow+\infty$ as $s \rightarrow+\infty$;

2. $u_{0}$ is suitably smooth (say $u_{0} \in C^{1}$ );

3. both $f(r)$ and $\varphi(r)$ are continuous and positive for $r$ in $(0,1)$.

The main result in this section is the following:

Theorem 1. Assume that $I(\lambda)=\infty$ for all positive constants $\lambda$. Then the problem

$$
\left\{\begin{array}{l}
\frac{\partial}{\partial t}\left(f(r) r^{n-1} u\right)=\frac{\partial}{\partial r}\left(r^{n-1} \varphi(1-r) \Psi\left(\frac{\partial u}{\partial r}\right)\right) \\
\left.J(r, t)\right|_{r=0}=0 \\
\left.u\right|_{t=0}=u_{0}(r)
\end{array}\right.
$$

has a unique bounded solution $u \in C_{*}^{1,1 / 2}\left(Q_{T}\right)$ such that $J(r, t) \in L^{\infty}([0, T], B V([0,1]))$.

To study the existence of the solutions to Problem (2.1), we first consider the regularized problem

$$
\begin{gathered}
\frac{\partial}{\partial t}\left(f_{\varepsilon}(r) r_{\varepsilon}^{n-1} u_{\varepsilon}\right)=\frac{\partial}{\partial r}\left(r_{\varepsilon}^{n-i} \varphi_{\varepsilon}(1-r) \Psi_{\varepsilon}\left(\frac{\partial u_{\varepsilon}}{\partial r}\right)\right) \\
\left.\frac{\partial u_{\varepsilon}}{\partial r}\right|_{r=0}=\left.\frac{\partial u_{\varepsilon}}{\partial r}\right|_{r=1}=0 \\
\left.u_{\varepsilon}\right|_{t=0}=u_{0}(r),
\end{gathered}
$$

where $r_{\varepsilon}=r+\varepsilon, f_{\varepsilon}=f+\varepsilon, \psi_{\varepsilon}=\psi+\varepsilon$, and $\varphi_{\varepsilon}=\varphi+\varepsilon$ with $\varepsilon$ a positive constant.

The classical results on initial boundary value problems to parabolic equations imply that the regularized problem (2.2)-(2.4) has a unique smooth solution for any given $\varepsilon>0$. We now start with some a priori estimates to the solutions of the regularized problem. We have

Lemma 2.1. There exists a constant $M$ independent of $\varepsilon$ such that the solution of $(2.2)-$ (2.4) satisfies the following estimates:

$$
\sup _{t \in[0, T]} \int_{0}^{1} r_{\varepsilon}^{n-1} f_{\varepsilon}(r)\left|\frac{\partial u_{\varepsilon}}{\partial t}\right| d r \leq M
$$

and

$$
\sup _{t \in[0, T]} \int_{0}^{1}\left|\frac{\partial J_{\varepsilon}}{\partial r}\right| d r \leq M
$$


where

$$
J_{\varepsilon}=r_{\varepsilon}^{n-1} \varphi_{\varepsilon}(1-r) \psi_{\varepsilon}\left(\left|\frac{\partial u_{\varepsilon}}{\partial r}\right|\right) \frac{\partial u_{\varepsilon}}{\partial r} .
$$

Proof. Differentiate (2.2) with respect to $t$ and set

Then

$$
v_{\varepsilon}=\frac{\partial u_{\varepsilon}}{\partial t} .
$$

$$
f_{\varepsilon}(r) \frac{\partial\left(r_{\varepsilon}^{n-1} v_{\varepsilon}\right)}{\partial t}=\frac{\partial}{\partial r}\left(r_{\varepsilon}^{n-1} \varphi_{\varepsilon}(1-r) \Psi_{\varepsilon}^{\prime}\left(\frac{\partial u_{\varepsilon}}{\partial r}\right) \frac{\partial v_{\varepsilon}}{\partial r}\right)
$$

Taking

$$
\eta_{\delta}(s)=\frac{s}{\sqrt{\delta+s^{2}}}
$$

and multiplying $(2.5)$ by $\eta_{\delta}\left(v_{\varepsilon}\right)$, we get

$$
\begin{aligned}
& \frac{\partial}{\partial t} \int_{0}^{1} f_{\varepsilon}(r) r_{\varepsilon}^{n-1} \sqrt{\delta+v_{\varepsilon}^{2}} d r \\
& \quad=\int_{0}^{1} \frac{\partial}{\partial r}\left(r_{\varepsilon}^{n-1} \varphi_{\varepsilon}(1-r) \Psi_{\varepsilon}^{\prime}\left(\frac{\partial u_{\varepsilon}}{\partial r}\right) \frac{\partial v_{\varepsilon}}{\partial r}\right) \eta_{\delta}\left(v_{\varepsilon}\right) d r
\end{aligned}
$$

That is,

$$
\begin{aligned}
& \frac{\partial}{\partial t} \int_{0}^{1} f_{\varepsilon}(r) r_{\varepsilon}^{n-1} \sqrt{\delta+v_{\varepsilon}^{2}} d r \\
& \quad=-\int_{0}^{1} r_{\varepsilon}^{n-1} \varphi_{\varepsilon}(1-r) \Psi_{\varepsilon}^{\prime}\left(\frac{\partial u_{\varepsilon}}{\partial r}\right)\left(\frac{\partial v_{\varepsilon}}{\partial r}\right)^{2} \eta_{\delta}^{\prime}\left(v_{\varepsilon}\right) d r
\end{aligned}
$$

Since all the functions in the integrand of the right-hand side integral are nonnegative and since $\left|\eta_{\delta}(s)\right| \leq 1$ for all $s$, we know that

$$
\frac{\partial}{\partial t} \int_{0}^{1} f_{\varepsilon}(r) r_{\varepsilon}^{n-1} \sqrt{\delta+v_{\varepsilon}^{2}} d r \leq 0
$$

Hence, the function in the left-hand side is nonincreasing in $t$,

$$
\begin{aligned}
\int_{0}^{1} f_{\varepsilon}(r) r_{\varepsilon}^{n-1} \sqrt{\delta+v_{\varepsilon}^{2}} d r \\
\quad \leq \int_{0}^{1} f_{\varepsilon}(r) r_{\varepsilon}^{n-1} \sqrt{\delta+v_{0}^{2}} d r \\
\quad \leq \int_{0}^{1}(f+1)(r+1)^{n-1}\left|\frac{\partial u_{0}}{\partial t}\right| d r+C
\end{aligned}
$$

with $C$ a constant independent of $\varepsilon<1$ and $\delta<1$. Letting $\delta \rightarrow 0$, we obtain

$$
\sup _{t \in[0, T]} \int_{0}^{1} f_{\varepsilon}(r) r_{\varepsilon}^{n-1}\left|\frac{\partial u_{\varepsilon}}{\partial t}\right| d r \leq M
$$

with $M$ independent of $\varepsilon$. We know from (2.2) and (2.6) that

$$
\sup _{t \in[0, T]} \int_{0}^{1}\left|\frac{\partial J_{\varepsilon}}{\partial r}\right| d r \leq M
$$

with $M$ independent of $\varepsilon \in(0,1)$ and $t$ in $[0, T]$. The lemma follows immediately.

To show the existence of weak solutions to the problem, we need the locally uniform Hölder estimates to the solutions of the regularized problem. We have 
LEMma 2.2. Under the assumptions of Theorem 1, the regularized solutions of Problem $(2.2),(2.3),(2.4)$ satisfy the following estimates:

$$
\left|J_{\varepsilon}\right| \leq M \quad \text { uniformly for }(x, t) \in Q_{T}
$$

and

$$
\left|\frac{\partial u_{\varepsilon}}{\partial r}\right| \leq M_{\delta}
$$

with $M$ as in (2.7) and $M_{\delta}$ depending only on the known quantities and $\delta$ uniformly for $(r, t) \in(\delta, 1-\delta) \times[0, T]$. Furthermore, for any $0<\Delta r<\delta / 2$, we have

$$
\left|u_{\varepsilon}(r+\Delta r, t)-u_{\varepsilon}(r, t)\right| \leq M_{\delta}|\Delta r|
$$

and

$$
\left|u_{\varepsilon}(r, t+\Delta t)-u_{\varepsilon}(r, t)\right| \leq M_{\delta}|\Delta t|^{1 / 2}
$$

if $t$ and $t+\Delta t$ are both in $[0, T], r \in(\delta, 1-\delta)$ with $M_{\delta}$ depending only on the quantities stated as above.

Proof. (2.8) is an immediate consequence of (2.7), and (2.9) can be easily derived from (2.8) by using the hypotheses on $\varphi$ and $\Psi$. (2.10) is obvious by (2.9). We have only to prove (2.11).

Note that for any $T \in[0, T], r \in(\delta, 1-\delta)$, and $\Delta t>0$ with $t+\Delta t \in[0, T],|\Delta t|<\delta / 2$,

$$
u_{\varepsilon}(r, t+\Delta t)-u_{\varepsilon}(r, t)=\int_{t}^{t+\Delta t} \frac{\partial u_{\varepsilon}}{\partial \tau}(r, \tau) d \tau
$$

Taking absolute value and integrating in $r$ over $\left(r, r+|\Delta t|^{1 / 2}\right)$, we get

$$
\begin{aligned}
\int_{r}^{r+|\Delta t|^{1 / 2}}\left|u_{\varepsilon}(s, t+\Delta t)-u_{\varepsilon}(x, t)\right| d s \\
\quad \leq \int_{r}^{r+|\Delta t|^{1 / 2}} \int_{t}^{t+\Delta t}\left|\frac{\partial u_{\varepsilon}}{\partial \tau}(s, \tau)\right| d \tau d s \\
\quad=\int_{t}^{t+\Delta t} \int_{r}^{r+|\Delta t|^{1 / 2}}\left|\frac{\partial u_{\varepsilon}}{\partial \tau}(s, \tau)\right| d s d \tau \\
\quad \leq|\Delta t| \sup _{\tau} \int_{\delta / 2}^{1-\delta / 2}\left|\frac{\partial u_{\varepsilon}}{\partial \tau}(s, \tau)\right| d s \\
\quad \leq C_{\delta}|\Delta t| \sup _{\tau} \int_{0}^{1} f_{\varepsilon}(r) r_{\varepsilon}^{n-1}\left|\frac{\partial u_{\varepsilon}}{\partial \tau}(s, \tau)\right| d s \leq M_{\delta}|\Delta t| .
\end{aligned}
$$

Here we have used (2.6) and the hypothesis on $f$. By applying the Mean Value Theorem for integrals, we may find an $r^{*} \in\left(r, r+|\Delta t|^{1 / 2}\right)$ such that

$$
\left|u_{\varepsilon}\left(r^{*}, t+\Delta t\right)-u_{\varepsilon}\left(r^{*}, t\right)\right| \leq M_{\delta}|\Delta t|^{1 / 2} .
$$


Hence, for any $(r, t) \in(\delta, 1-\delta) \times[0, T]$,

$$
\begin{aligned}
\mid u_{\varepsilon}(r, & +\Delta t)-u_{\varepsilon}(r, t) \mid \\
\leq & \left|u_{\varepsilon}(r, t+\Delta t)-u_{\varepsilon}\left(r^{*}, t+\Delta t\right)\right|+\left|u_{\varepsilon}\left(r^{*}, t+\Delta t\right)-u_{\varepsilon}\left(r^{*}, t\right)\right| \\
& +\left|u_{\varepsilon}\left(r^{*}, t\right)-u_{\varepsilon}(r, t)\right| \\
\leq & M_{\delta}|\Delta t|^{1 / 2} .
\end{aligned}
$$

This is what we need. The proof is complete.

Proof of Theorem 1. Combining (2.10) and (2.11), we obtain

$$
\left|u_{\varepsilon}(r+\Delta r, t+\Delta t)-u_{\varepsilon}(r, t)\right| \leq M_{\delta}\left(|\Delta r|+|\Delta t|^{1 / 2}\right)
$$

for all $(r, t) \in(\delta, 1-\delta) \times[0, T]$ uniformly with $M_{\delta}$ independent of $\varepsilon$. By the Ascoli-Arzela Theorem, there exists a function $u \in C_{*}^{1,1 / 2}$ such that

$$
u_{\varepsilon}(r, t) \rightarrow u(r, t) \quad \text { uniformly in }(\delta, 1-\delta) \times[0, T] .
$$

(2.8) and (2.9) imply that

$$
\frac{\partial u_{\varepsilon}}{\partial r} \rightarrow \frac{\partial u}{\partial r} \text { in } L_{\mathrm{loc}}^{\infty} \text { weak }^{*} \text { topology. }
$$

To show that $u$ is a solution of $(2.2)$, is suffices to prove that for any $\phi \in C_{0}^{\infty}\left(Q_{T}\right)$,

$$
\iint_{Q_{T}}\left(f(r) r^{n-1} u(r, t) \frac{\partial \phi}{\partial t}-r^{n-1} \varphi(1-r) \Psi\left(\frac{\partial u}{\partial r}\right) \frac{\partial \phi}{\partial r}\right) d r d t=0 .
$$

By the definition of $u_{\varepsilon}$, the above identity holds for $u_{\varepsilon}$, i.e.,

$$
\iint_{Q_{T}}\left(f_{\varepsilon}(r) r_{\varepsilon}^{n-1} u_{\varepsilon}(r, t) \frac{\partial \phi}{\partial t}-r_{\varepsilon}^{n-1} \varphi_{\varepsilon}(1-r) \Psi_{\varepsilon}\left(\frac{\partial u_{\varepsilon}}{\partial r}\right) \frac{\partial \phi}{\partial r}\right) d r d t=0
$$

Since $u_{\varepsilon} \rightarrow u$ pointwise in the supporting set, $\operatorname{supp} \phi$, of $\phi$, we know that it suffices to prove that

$$
\lim _{\varepsilon \rightarrow 0} \iint_{Q_{T}} r_{\varepsilon}^{n-1} \varphi_{\varepsilon}(1-r) \Psi_{\varepsilon}\left(\frac{\partial u_{\varepsilon}}{\partial r}\right) \frac{\partial \phi}{\partial r} d r d t
$$

exists and is equal to

$$
\iint_{Q_{T}} r^{n-1} \varphi(1-r) \Psi\left(\frac{\partial u}{\partial r}\right) \frac{\partial \phi}{\partial r} d r d t
$$

Let us first multiply $(2.2)$ by $\left(u_{\varepsilon}-u\right) \phi$ and integrate over $Q_{T}$. Then

$$
\iint_{Q_{T}} \frac{\partial}{\partial t}\left(f_{\varepsilon}(r) r_{\varepsilon}^{n-1} u_{\varepsilon}\right)\left(u_{\varepsilon}-u\right) \phi d r d t=-\iint_{Q_{T}} J_{\varepsilon} \frac{\partial}{\partial r}\left[\left(u_{\varepsilon}-u\right) \phi\right] d r d t
$$


Notice that

$$
\begin{aligned}
& \iint_{Q_{T}} \frac{\partial}{\partial t}\left(f_{\varepsilon}(r) r_{\varepsilon}^{n-1} u_{\varepsilon}\right)\left(u_{\varepsilon}-u\right) \phi d r d t \\
& \quad \leq C \sup _{(r, t) \in \operatorname{supp} \phi}\left|u_{\varepsilon}-u\right| \iint_{Q_{T}}\left|\frac{\partial u_{\varepsilon}}{\partial t}\right| d r d t \rightarrow 0
\end{aligned}
$$

as $\varepsilon \rightarrow 0$ for fixed $\phi \in C_{0}^{\infty}\left(Q_{T}\right)$, where $C$ is a constant depending only on the maximum of $f$. Furthermore,

$$
\begin{aligned}
& \iint_{Q_{T}} J_{\varepsilon} \frac{\partial}{\partial r}\left[\left(u_{\varepsilon}-u\right) \phi\right] d r d t \\
& \quad=\iint_{Q_{T}} J_{\varepsilon}\left(u_{\varepsilon}-u\right) \frac{\partial \phi}{\partial r} d r d t+\iint_{Q_{T}} J_{\varepsilon}\left(\frac{\partial u_{\varepsilon}}{\partial r}-\frac{\partial u}{\partial r}\right) \phi d r d t
\end{aligned}
$$

It is clear that the integrand of the first integral on the right-hand side of $(2.17)$ is bounded since $J_{\varepsilon}$ is uniformly bounded, $u_{\varepsilon}$ and $u$ are bounded on $\operatorname{supp} \phi$ by Lemma 2.2, and the Lebesgue Dominated Theorem shows that the first integral goes to 0 as $\varepsilon \rightarrow 0$. Then (2.15), (2.16), and (2.17) imply that the last term on the right-hand side of (2.17) also goes to 0 .

We write

$$
\begin{aligned}
\iint_{Q_{T}} J_{\varepsilon} & \left(\frac{\partial u_{\varepsilon}}{\partial r}-\frac{\partial u}{\partial r}\right) \phi d r d t \\
= & \iint_{Q_{T}} r_{\varepsilon}^{n-1} \varphi_{\varepsilon}(1-r)\left[\Psi_{\varepsilon}\left(\frac{\partial u_{\varepsilon}}{\partial r}\right)-\Psi_{\varepsilon}\left(\frac{\partial u}{\partial r}\right)\right]\left(\frac{\partial u_{\varepsilon}}{\partial r}-\frac{\partial u}{\partial r}\right) \phi \\
& +\iint_{Q_{T}}\left\{r_{\varepsilon}^{n-1} \varphi_{\varepsilon}(1-r) \Psi_{\varepsilon}\left(\frac{\partial u}{\partial r}\right)-r^{n-1} \varphi(1-r) \Psi\left(\frac{\partial u}{\partial r}\right)\right\}\left(\frac{\partial u_{\varepsilon}}{\partial r}-\frac{\partial u}{\partial r}\right) \phi \\
& +\iint_{Q_{T}} r^{n-1} \varphi(1-r) \Psi\left(\frac{\partial u}{\partial r}\right)\left(\frac{\partial u_{\varepsilon}}{\partial r}-\frac{\partial u}{\partial r}\right) \phi
\end{aligned}
$$

It is obvious that

$$
\lim _{\varepsilon \rightarrow 0} r_{\varepsilon}^{n-1} \varphi_{\varepsilon}(1-r) \Psi_{\varepsilon}\left(\frac{\partial u}{\partial r}\right)=r^{n-1} \varphi(1-r) \Psi\left(\frac{\partial u}{\partial r}\right),
$$

for any $(r, t) \in \operatorname{supp} \phi$. Since $\left|\frac{\partial u_{\varepsilon}}{\partial r}\right|\left|\frac{\partial u}{\partial r}\right|$ are bounded on $\operatorname{supp} \phi$ independently of $\varepsilon$, Lebesgue's Dominated Convergence Theorem shows that the second term of the righthand side of (2.18) tends to 0 as $\varepsilon \rightarrow 0$. (2.14) then implies that the last term of the right-hand side of $(2.18)$ goes to 0 as $\varepsilon \rightarrow 0$.

Therefore, by (2.18),

$$
\lim _{\varepsilon \rightarrow 0} \iint_{Q_{T}} r_{\varepsilon}^{n-1} \varphi_{\varepsilon}(1-r)\left[\Psi_{\varepsilon}\left(\frac{\partial u_{\varepsilon}}{\partial r}\right)-\Psi_{\varepsilon}\left(\frac{\partial u}{\partial r}\right)\right]\left(\frac{\partial u_{\varepsilon}}{\partial r}-\frac{\partial u}{\partial r}\right) \phi d r d t=0 .
$$

It is easily seen by checking the proofs as above that (2.19) is still true if we replace $\phi \in C_{0}^{\infty}\left(Q_{T}\right)$ by $|\phi|$ since we only used the properties of $\phi$ having compact support and 
$\frac{\partial \phi}{\partial r}$ being bounded. Therefore, we denote $\omega_{\varepsilon}=\frac{\partial u_{\varepsilon}}{\partial r}$ and $\omega=\frac{\partial u}{\partial r}$. Then

$$
\begin{aligned}
\left|\iint_{Q_{T}} r_{\varepsilon}^{n-1} \varphi_{\varepsilon}(1-r)\left[\Psi_{\varepsilon}\left(\omega_{\varepsilon}\right)-\Psi_{\varepsilon}(\omega)\right] \phi d r d t\right| \\
=\left|\iint_{Q_{T}} r_{\varepsilon}^{n-1} \varphi_{\varepsilon}(1-r)\left[\int_{0}^{1} \Psi_{\varepsilon}^{\prime}\left(\tau w_{\varepsilon}+(1-\tau) w\right) d \tau\right]\left(\omega_{\varepsilon}-\omega\right) \phi d r d t\right| \\
\leq \\
\quad\left(\iint_{Q_{T}} r_{\varepsilon}^{n-1} \varphi_{\varepsilon}(1-r)\left[\int_{0}^{1} \Psi_{\varepsilon}^{\prime}\left(\tau w_{\varepsilon}+(1-\tau) w\right) d \tau\right]|\phi| d r d t\right)^{1 / 2} \\
\quad \times\left(\iint_{Q_{T}} r_{\varepsilon}^{n-1} \varphi_{\varepsilon}(1-r)\left[\int_{0}^{1} \Psi_{\varepsilon}^{\prime}\left(\tau w_{\varepsilon}+(1-\tau) w\right) d \tau\right]\left(\omega_{\varepsilon}-\omega\right)^{2}|\phi| d r d t\right)^{1 / 2} \\
\leq C\left(\iint_{Q_{T}} r_{\varepsilon}^{n-1} \varphi_{\varepsilon}(1-r)\left[\Psi_{\varepsilon}\left(w_{\varepsilon}\right)-\Psi_{\varepsilon}(\omega)\right]\left(w_{\varepsilon}-\omega\right)|\phi| d r d t\right)^{1 / 2} \rightarrow 0
\end{aligned}
$$

as $\varepsilon \rightarrow 0$ by (2.19). Since the functions in the integrand of the first integral in the product are all bounded uniformly on $\operatorname{supp} \phi$, the first term in the product is bounded independently of $\varepsilon \in(0,1)$.

Combining all the results in this proof, we know that $u$ is a solution of $(2.1)$ in the sense of distribution and obviously it satisfies the regularity requirements such as $u \in C_{*}^{1,1 / 2}$ and $J \in L^{\infty}([0, T], B V([0,1]))$ in Theorem 1 .

The boundary-value and the initial-value conditions can be easily checked by examining the proofs and the uniform estimates as above. The boundedness of the solution can be easily shown by applying Theorem 14 on p. 49 of [6] to the solution of $(2.2)-(2.4)$. We omit the details.

Therefore, the existence of solutions to Problem (2.1) is clear. We have only to show the uniqueness of the solution. Since $J(r, t) \in L^{\infty}([0, T], B V([0,1]))$, the limit

$$
\lim _{r \rightarrow 1} J(r, t)=l(t)
$$

exists for almost all $t \in[0, T]$. We claim that $l(t)=0$, a.e. $t \in(0, T)$. If this were not true, without loss of generality, we might assume that for some $t_{0} \in[0, T], \lim _{r \rightarrow 1} J\left(r, t_{0}\right)=$ $l\left(t_{0}\right)$ exists and $l\left(t_{0}\right)>0$. Then there would be an $\eta>0$ such that

$$
J\left(r, t_{0}\right)>\frac{l\left(t_{0}\right)}{2} \quad \text { for all } r \in(1-\eta, 1)
$$

Therefore, by the definition of $J$,

$$
\begin{aligned}
u\left(r_{2}, t_{0}\right)-u\left(r_{1}, t_{0}\right) & =\int_{r_{1}}^{r_{2}} \Psi^{-1}\left(\frac{J\left(r, t_{0}\right)}{r^{n-1} \varphi(1-r)}\right) d r \\
& \geq \int_{r_{1}}^{r_{2}} \Psi^{-1}\left(\frac{l\left(t_{0}\right) 2^{n-2}}{\varphi(1-r)}\right) d r
\end{aligned}
$$

This contradicts the boundedness of the function $u$ since the last integral is unbounded by the assumptions. The claim is proven. 
Now, assume that $u_{1}$ and $u_{2}$ are two solutions of Problem (2.1). Set $z=u_{1}-u_{2}$ and

$$
J_{i}=r^{n-1} \varphi(1-r) \Psi\left(\frac{\partial u_{i}}{\partial r}\right)
$$

for $i=1,2$. Then

$$
\left\{\begin{array}{l}
\frac{\partial}{\partial t}\left(f(r) r^{n-1} z\right)=\frac{\partial}{\partial r}\left(J_{1}-J_{2}\right), \\
\left.J_{i}(r, t)\right|_{r=0}=\left.J_{i}(r, t)\right|_{r=1}=0, \quad \text { for } i=1,2 \\
\left.z\right|_{t=0}=u_{0}(r)
\end{array}\right.
$$

Set

$$
H_{\eta}(s)=\frac{s}{\sqrt{\eta+s^{2}}}, \quad \mathcal{H}_{\eta}(s)=\int_{0}^{s} H_{\eta}(\sigma) d \sigma .
$$

Multiplying the first equation in (2.21) with $H_{\eta}(z)$ and using the boundary conditions, writing $\omega_{i}=\frac{\partial u_{i}}{\partial r}$ for $i=1,2$, we get

$$
\begin{array}{rl}
\frac{\partial}{\partial t} \int_{0}^{1} & f(r) r^{n-1} \mathcal{H}_{\eta}(z) d r \\
\quad= & \int_{0}^{1} H_{\eta}(z) \frac{\partial}{\partial r}\left(J_{1}-J_{2}\right) d r \\
& =-\int_{0}^{1} H_{\eta}^{\prime}(z) \frac{\partial z}{\partial r}\left(J_{1}-J_{2}\right) d r \\
& =-\int_{0}^{1} H_{\eta}^{\prime}(z) r^{n-1} \varphi\left(\Psi\left(\omega_{1}\right)-\Psi\left(\omega_{2}\right)\right) \times\left(\omega_{1}-\omega_{2}\right) d r \leq 0 .
\end{array}
$$

This implies that

$$
\begin{array}{rl}
\int_{0}^{1} & f(r) r^{n-1} \mathcal{H}_{\eta}(z(r, t)) d r \\
& \leq \int_{0}^{1} f(r) r^{n-1} \mathcal{H}_{\eta}\left(\left.z\right|_{t=0}\right) d r \\
& =\int_{0}^{1} f(r) r^{n-1} \frac{\sqrt{\eta}}{n} d r .
\end{array}
$$

Letting $\eta \rightarrow 0$, we know that $f(r) r^{n-1} z(r, t) \equiv 0$ for all $(r, t) \in Q_{T}$. The uniqueness is proven.

The proof of Theorem 1 is complete.

REMARK 1. Note that in the hypotheses, we assume the $C^{1}$ smoothness of the function $\Psi$. By carefully checking the proof, it is not difficult to see that Theorem 1 holds if we replace the smoothness of $\Psi$ by the condition that either $\Psi \in C^{1}$ or $\Psi^{-1} \in C^{1}$. In fact, this condition was used in the proof of $(2.20)$ and there will be no substantial difficulties in proving the same estimate if we use $\Psi^{-1} \in C^{1}$ to replace the original one.

REMARK 2. If we use the equation of non-Newtonian electric filtration to replace the equation in (2.1), then there is no isolated environment because of the special structure of the equation for which $I(\lambda)<\infty$ for any constant $\lambda$. Such cases will be discussed in the next section. 


\section{Existence of the state depending on both the initial and the boundary} conditions. In the second section, we have that a sufficient condition to have an isolated environment is that $I(\lambda)=\infty$ for any constant $\lambda$. Then, a natural problem is what will be the case if $I(\lambda)<\infty$ for some constant $\lambda$ ? We shall partially answer this question in the present section. The main result in this section is the following.

Theorem 2. Assume that $I(\lambda)<\infty$ for any positive constant $\lambda$. Then, if $g \in C^{2}$, the problem

$$
\left\{\begin{array}{l}
\frac{\partial}{\partial t}\left(f(r) r^{n-1} u\right)=\frac{\partial}{\partial r}\left(r^{n-1} \varphi(1-r) \Psi\left(\frac{\partial u}{\partial r}\right)\right) \\
\left.J(r, t)\right|_{r=0}=0,\left.\quad u\right|_{r=1}=g(t) \\
\left.u\right|_{t=0}=u_{0}(r)
\end{array}\right.
$$

has a unique solution $u$ in $C_{*}^{1,1 / 2}\left(Q_{T}\right)$ and $J \in L^{\infty}([0, T], B V([0,1]))$.

This result shows that if $I(\lambda)<\infty$ for any positive constant $\lambda$, then the state of the flow depends on the surrounding environment; furthermore, the state is uniquely determined by both the boundary behavior and the initial state.

If $w$ is a solution of the above problem and if we let $u=w-g$, then

$$
f(r) \frac{\partial\left(r^{n-1} u\right)}{\partial t}=\frac{\partial}{\partial r}\left(r^{n-1} \varphi(1-r) \psi\left(\left|\frac{\partial u}{\partial r}\right|\right) \frac{\partial u}{\partial r}\right)+f(r) r^{n-1} g^{\prime}(t) .
$$

The corresponding initial and boundary conditions are

$$
\left.u\right|_{t=0}=u_{0}(r)-g(0),\left.\quad u\right|_{r=1}=0
$$

and

$$
\left.J\right|_{r=0}=0
$$

Then, the proof of Theorem 2 is reduced to proving that under the hypotheses of Theorem 2, Problem (3.1)-(3.3) has a unique solution.

Without loss of generality, we may assume that $g(0)=0$ in the following.

To study the existence of solutions to the problem (3.1)-(3.3), we first study the regularized problem

$$
f_{\varepsilon}(r) \frac{\partial\left(r_{\varepsilon}^{n-1} u_{\varepsilon}\right)}{\partial t}=\frac{\partial}{\partial t}\left(r_{\varepsilon}^{n-1} \varphi_{\varepsilon}(1-r) \psi_{\varepsilon}\left(\left|\frac{\partial u_{\varepsilon}}{\partial r}\right|\right) \frac{\partial u_{\varepsilon}}{\partial r}\right)+f_{\varepsilon}(r) r_{\varepsilon}^{n-1} g^{\prime}(t)
$$

The initial and boundary conditions are (3.2) and

$$
\left.\frac{\partial u_{\varepsilon}}{\partial r}\right|_{r=0}=0
$$

As in the second section, the classical results on initial boundary-value problems to parabolic equations imply that the regularized problem $\left(3.1^{\prime}\right),(3.2),\left(3.3^{\prime}\right)$ has a unique smooth solution for any given $\varepsilon>0$.

Also, as in the previous section, we have the following a priori estimates to the solution of the regularized problem. 
LEMMA 3.1. There exists a constant $M$ independent of $\varepsilon$ such that the solution of $\left(3.1^{\prime}\right),(3.2),\left(3.3^{\prime}\right)$ satisfies the following estimates:

$$
\sup _{t \in[0, T]} \int_{0}^{1} r_{\varepsilon}^{n-1} f_{\varepsilon}(r)\left|\frac{\partial u_{\varepsilon}}{\partial t}\right| d r \leq M
$$

and

$$
\sup _{t \in[0, T]} \int_{0}^{1}\left|\frac{\partial J_{\varepsilon}}{\partial r}\right| d r \leq M
$$

Proof. As in the proof of Lemma 2.1, differentiate $\left(3.1^{\prime}\right)$ with respect to $t$ and set

$$
v_{\varepsilon}=\frac{\partial u_{\varepsilon}}{\partial t} .
$$

Then,

$$
f_{\varepsilon}(r) \frac{\partial\left(r_{\varepsilon}^{n-1} v_{\varepsilon}\right)}{\partial t}=\frac{\partial}{\partial r}\left(r_{\varepsilon}^{n-1} \varphi_{\varepsilon}(1-r) \Psi_{\varepsilon}^{\prime}\left(\frac{\partial u_{\varepsilon}}{\partial r}\right) \frac{\partial v_{\varepsilon}}{\partial r}\right)+f_{\varepsilon}(r) r_{\varepsilon}^{n-1} g^{\prime \prime}(t) .
$$

Taking $\eta_{\delta}(s)$ as before and multiplying (3.4) by $\eta_{\delta}\left(v_{\varepsilon}\right)$, we get

$$
\begin{aligned}
\frac{\partial}{\partial t} \int_{0}^{1} f_{\varepsilon}(r) r_{\varepsilon}^{n-1} \sqrt{\delta+v_{\varepsilon}^{2}} d r+\int_{0}^{1} r_{\varepsilon}^{n-1} \varphi_{\varepsilon}(1-r) \Psi_{\varepsilon}^{\prime} & \left(\frac{\partial u_{\varepsilon}}{\partial r}\right)\left(\frac{\partial v_{\varepsilon}}{\partial r}\right)^{2} \eta_{\delta}^{\prime}\left(v_{\varepsilon}\right) d r \\
& =\int_{0}^{1} f_{\varepsilon}(r) r_{\varepsilon}^{n-1} g^{\prime \prime}(t) \eta_{\delta}\left(v_{\varepsilon}\right) d r
\end{aligned}
$$

Reasoning as in Sec. 2,

$$
\frac{\partial}{\partial t} \int_{0}^{1} f_{\varepsilon}(r) r_{\varepsilon}^{n-1} \sqrt{\delta+v_{\varepsilon}^{2}} d r \leq \int_{0}^{1} f_{\varepsilon}(r)(r+1)^{n-1}\left|g^{\prime \prime}(t)\right| d r \leq C
$$

with $C$ independent of $\varepsilon$ and $u_{\varepsilon}$.

Hence, integrating with respect to $t$, we have

$$
\begin{aligned}
& \int_{0}^{1} f_{\varepsilon}(r) r_{\varepsilon}^{n-1} \sqrt{\delta+v_{\varepsilon}^{2}} d r \\
& \quad \leq C T+\int_{0}^{1} f_{\varepsilon}(r) r_{\varepsilon}^{n-1} \sqrt{\delta+v_{0}^{2}} d r .
\end{aligned}
$$

Letting $\delta \rightarrow 0$, we obtain

$$
\sup _{t \in[0, T]} \int_{0}^{1} f_{\varepsilon}(r) r_{\varepsilon}^{n-1}\left|\frac{\partial u_{\varepsilon}}{\partial t}\right| d r \leq M
$$

with $M$ independent of $\varepsilon$. The lemma then follows as in Sec. 2 .

The statement and the proof of the locally uniform Hölder estimates to the solutions of the regularized problem are exactly the same as in Lemma 2.2 of Sec. 2. We will simply use the results without proof. 
Proof of Theorem 2. With Lemma 3.1, Lemma 2.2, and using the same arguments as in proving the existence of solutions to Problem (2.1), we can easily know that Problem (3.1) has a weak solution $u$ in the sense of distribution, and also the solution satisfies the regularity requirements in Theorem 2 .

We need further to prove that $u$ satisfies the initial and boundary conditions (3.2) and (3.3).

By definition,

$$
J_{\varepsilon}=r_{\varepsilon}^{n-1} \varphi_{\varepsilon}(1-r) \Psi_{\varepsilon}\left(\frac{\partial u_{\varepsilon}}{\partial r}\right)
$$

Then, since $\Psi_{\varepsilon}$ is monotonically increasing and therefore has an inverse, we get

$$
\frac{\partial u_{\varepsilon}}{\partial r}=\Psi_{\varepsilon}^{-1}\left(\frac{J_{\varepsilon}}{r_{\varepsilon}^{n-1} \varphi_{\varepsilon}(1-r)}\right) .
$$

Integrating both sides of Eq. (3.6) with respect to $r$, we have

$$
u_{\varepsilon}\left(r_{2}, t\right)-u_{\varepsilon}\left(r_{1}, t\right)=\int_{r_{1}}^{r_{2}} \Psi_{\varepsilon}^{-1}\left(\frac{J_{\varepsilon}}{r_{\varepsilon}^{n-1} \varphi_{\varepsilon}(1-r)}\right) d r .
$$

Since $\Psi_{\varepsilon}$ is an odd function, so is $\Psi_{\varepsilon}^{-1}$. Therefore for $r_{2}>r_{1}>1 / 2$,

$$
\begin{aligned}
\left|u_{\varepsilon}\left(r_{2}, t\right)-u_{\varepsilon}\left(r_{1}, t\right)\right| & \\
& \leq \int_{r_{1}}^{r_{2}} \Psi_{\varepsilon}^{-1}\left(\frac{\left|J_{\varepsilon}\right|}{r_{\varepsilon}^{n-1} \varphi_{\varepsilon}(1-r)}\right) d r \\
& \leq \int_{r_{1}}^{r_{2}} \Psi^{-1}\left(\frac{M}{r^{n-1} \varphi(1-r)}\right) d r \\
& \leq \int_{r_{1}}^{r_{2}} \Psi^{-1}\left(\frac{2^{n-1} M}{\varphi(1-r)}\right) d r
\end{aligned}
$$

where we used the estimate in Lemma 2.2 and the monotonicity of the function $\Psi$. Letting $r_{2} \rightarrow 1$ and using the boundary condition (3.2), we have

$$
\left|u_{\varepsilon}\left(r_{1}, t\right)\right| \leq \int_{r_{1}}^{1} \Psi^{-1}\left(\frac{M_{1}}{\varphi(1-r)}\right) d r .
$$

Letting $\varepsilon \rightarrow 0$, we know that

$$
\left|u\left(r_{1}, t\right)\right| \leq \int_{r_{1}}^{1} \Psi^{-1}\left(\frac{M_{1}}{\varphi(1-r)}\right) d r
$$

uniformly for $r_{1} \in(1 / 2,1)$. The assumption on $I(\lambda)$ implies that $u(r, t) \rightarrow 0$ uniformly in $t$ as $r \rightarrow 1$.

The other initial and boundary conditions in (3.2) and (3.3) can be shown easily by checking the previous proofs of the theorems. The uniqueness follows from an analogous argument in proving the uniqueness result in Theorem 1. We omit the details.

The proof of Theorem 2 is then easily done by letting $U(r, t)=u(r, t)+g(t)$. The proof is complete.

By carefully checking the proof of Theorem 2, we can easily prove the following Theorem. 
Theorem 3. Assume that $I(\lambda)<\infty$ for some positive constant $\lambda$. Then the problem stated in Theorem 2 has a unique solution $u$ in $C_{*}^{1,1 / 2}\left(Q_{T}\right)$ if

$$
\begin{aligned}
& T \sup _{t \in[0, T]}\left|g^{\prime \prime}(t)\right| \int_{0}^{1}(r+1)^{n-1}(f(r)+1) d r \\
& \quad+\int_{0}^{1}(r+1)^{n-1}(f(r)+1)\left|\frac{\partial u_{0}}{\partial t}\right| d r \leq \lambda .
\end{aligned}
$$

Furthermore, $J \in L^{\infty}([0, T], B V([0,1]))$.

Proof. The result follows by checking the proof of inequality (2.7) in the second section. We omit the details.

REMARK 3. Our results show that the integral $I(\lambda)$ plays an important role in determining whether the state of the gas or fluid may depend on the surrounding environment. Our results depend on the special structure of the equation and it is not difficult to generalize these results to some other special cases. But the problem is rather special in that the domain of definition is a ball and the spatial dependence of the porosity, and the quotient, permeability/viscosity, is purely radial. This can be regarded as a first step in the treatment of a class of porous medium problems. There are still some problems remaining unsolved such as $\phi(x, t, u, \nabla u)$ taking a general form or if the conditions in Theorem 3 are a sufficient and necessary condition, etc. These may result in our further work or the work of interested readers.

Acknowledgment. We thank the editor and the referee for pointing out some errors we made in the first version of this paper.

\section{REFERENCES}

[1] N. Ahmed and D. K. Sunada, Nonlinear flows in porous media. J. Hydraulics Div. Proc. Amer. Soc. Civil Eng. 95, 1847-1857 (1969)

[2] D. G. Aronson, L. A. Caffarelli, and S. Kamin, How an initially stationary interface begins to move in porous medium flow. Univ. of Minnesota Math. Report 81-113, 141 (1981)

[3] L. A. Caffarelli and A. Friedman, Regularity of the free boundary of a gas flow in an n-dimensional porous medium, Indiana Univ. Math. J. 29, $361389(1980)$

[4] L. A. Caffarelli, J. L. Vazquez, and N. I. Wolanski, Lipschitz continuity of solutions and interfaces of the $N$-dimensional porous medium equation. Indiana Univ. Math. J. 36(2), 327-401 (1987)

[5] J. R. Esteban and J. L. Vazquez, On the equation of turbulent filtration in one-dimensional porous media, Nonlinear Anal. T. M. A. 10, 13031325 (1986)

[6] A. Friedman, Partial Differential Equations of Parabolic: Type, Prentice-Hall, Inc., 1964

[7] A. Friedman and S. Kamin, The asymptotic behavior of gas in an $n$-dimensional porous medium. Trans. Amer. Nath. Soc. 262, 551563 (1980)

[8] B. H. Gilding, Properties of solutions of an equation in the theory of infiltration, Arch. Rational Mech. Anal. 65. no. 3. 203225 (1977)

[9] A. S. Kalashnikov, Some problems of the qualitative theory of nonlinear degenerate second-order parabolic equations, Russian Math. Surveys 42:2, $169-222$ (1987)

[10] L. K. Martinson and K. B. Paplov, The effect of magnetic plasticity in non-Newtonian fluids. Magnit. Gidrodinamika 3. 69- 75 (1969)

[11] P. Z. Mkrtychyan, Uniqueness of the solution to the second initial-boundary value problem for an equation of non-Neutonian polytropic filtration. (Russian) Zap. Nauchn. Sem. S.-Peterberg Otdel. Mat. Inst. Steklov (POMI) 200 (1992); Kraev Zadachi Mat. Fiz. Smezh Voprosy Teor. Funktsii 24, $110-117,189$

[12] J. R. King, Multidimensional singular diffusion. J. Engrg. Math. 27. 357387 (1993) 